\title{
Evaluation of methods for the estimation of mutation rates in cultured mammalian cell populations*
}

\author{
I-chian $\mathrm{Li}$ and E.H.Y. Chu \\ Department of Medicine, Medical College, National Cheng Kung University, Tainan 70101 (Taiwan) and Department of Human \\ Genetics, Medical School, University of Michigan, Ann Arbor, MI 48109 (U.S.A.)
}

(Accepted 29 November 1986)

Keywords: Mutation rates, evaluation; Mammalian cell populations, cultured; Statistical methods; (Cultured Chinese hamster cells V79); Fluctuation tests.

\section{Summary}

A systematic comparison of 5 different statistical methods for the estimation of mutation rate $(\mu)$ in cultured Chinese hamster V79 cells is presented. Fluctuation tests were performed with several large batches of parallel cell cultures each allowed to grow for a different length of time in order to reach different population size $\left(N_{t}\right)$. Based on Lea and Coulson's theoretical distribution, a comparison has been made between the experimental data and the expected distribution of the number of ouabain-resistant mutants per culture in these hamster cell populations. The sum of squared deviation between the observed and expected values, or SSD, was used as a means of the adequacy of the estimation method; the method which gives the smallest SSD is regarded as the best one for the estimation of $\mu$. Our results show that when $N_{t}$ is small, the occurrence of mutation is infrequent, and SSDs from different methods are similar. However, when $N_{\mathrm{t}}$ is large, there is a great discrepancy of the SSD values, suggesting a preference of using the maximum likelihood method, the $P_{0}$ method, the median method, the upper quartile method and the mean method, in that order, for the estimation of $\mu$. The order of preference is correlated with estimation efficiencies. Depending on the size of $N_{\mathrm{t}}$ and the method used, the estimated $\mu$ may vary up to more than 3 -fold. At a large $N_{\mathrm{t}}$, the $\mu$ obtained from the maximum likelihood method is very precise. This suggests the importance of choosing an appropriate $N_{\mathrm{t}}$ as well as method for the estimation of $\mu$.

There is considerable variation of the estimates $(\hat{\mu})$ of mutation rates in cultured mammalian cells when the cell populations are analyzed by the commonly used method of Luria and Delbrück's

\footnotetext{
* Supported in part by a grant from the National Science Council 74-0412-B006-13.

Correspondence: Dr. I-chian Li, Department of Medicine, Medical College, National Cheng Kung University, Tainan 70101 (Taiwan).
}

(1943) fluctuation analysis. The variation may have resulted from (a) possible differential mutability present in different cell lines, (b) different statistical methods used for the estimation of the mutation rates $(\mu)$, (c) various experimental designs followed by investigators, or (d) some other experimental errors. We ( $\mathrm{Li}$ et al., 1983) have elsewhere reported a systematic appraisal of the factors that may affect $\hat{\mu}$; we found that the number of parallel cultures and the final popula- 
tion size do affect the variance of $\hat{\mu}$, and hence the reliability of $\hat{\mu}$.

Based on Luria and Delbrück's stochastic approach for the estimation of $\mu$, Lea and Coulson (1949) had derived a statistical distribution for the numbers of mutants $(y)$ in a culture of bacteria in which the number of mutants increases both as a result of new mutations and cell division of old mutants. The distribution of $y$ is expressed in terms of only one parameter, $m$, the number of mutations expected in cultures growing to a population size $N_{\mathrm{t}}$. There is a differential realization of mutation in cell populations with various sizes; the larger the population size, the higher the number of mutation. Hence, the distribution of $y$ changes according to the final population size $\left(N_{t}\right)$.

Mutation rate in general is obtained by first estimating $m$ via one of a number of alternative methods, then dividing $m$ by $N_{\mathrm{t}}$. The problem arising in the estimation of $\mu$ from an observed distribution of $y$ in a series of cultures stems from the discrepancy of estimating $m$ by different methods. Out of the 5 available methods for the estimation of $m$, the mean method is widely used, the $P_{0}$ method and the median method are used to some extent, and the upper quartile method and the maximum likelihood method are never tried, as far as we are aware. However, contrary to the prevailing application, the maximum likelihood method is statistically the best method and the mean method probably is the worst one (see below) to follow for the estimation of $m$. We therefore make the first effort ever made in cultured mammalian cells by systematically comparing the estimation methods of $m$ in Chinese hamster V79 cells, using ouabain resistance as the mutation marker. The cell population was grown to various sizes and the change of the distribution pattern of $y$ (the ouabain-resistant colonies) was observed. Since the expected distribution of $y$ varies according to $m$, which is in turn affected by the estimation method, the method that mostly matches the observed and the expected distribution of $y$ is to be preferred. The statistic of the sum of squared deviation (SSD) between the observed and the expected values of $y$ is used as an indicator of the match. The aim is to find the method that gives the smallest SSD, the best match. This method is also expected to have the best estimation efficiency.

The present study represents our continuous effort to investigate the mutation rate in cultured mammalian cells (Fu et al., 1982; Li et al., 1983, 1985).

\section{Materials and methods}

Chinese hamster V79 cells were maintained in Eagle's modified minimum essential medium supplemented with 1.5-fold of nonessential amino acids, 1.5 -fold of vitamins, and $1 \mathrm{mM}$ of sodium pyruvate. 4 fluctuation experiments were established, each with 20 parallel cultures. The cell populations for each experiment were initiated by inoculating 100 cells per parallel culture into growth medium. The cells were allowed to grow for different lengths of time for different experiments. To minimize the variation of growth rate between experiments and within each 20 parallel cultures, the growth conditions for the experiment were set to be identical. Cell population was maintained at exponential growth via regular cell dispersion. The time spent to disperse the cells in the first and the last culture was made as short as possible, usually within $2 \mathrm{~h}$. The cell dispersion was done when the cell colonies in the culture dish had reached an appropriate size with each cell being quite healthy or when the culture had reached about $90 \%$ confluence. At the time of cell dispersion, the total number of cells in each culture was also recorded. The cells were then removed from the culture dishes by trypsinization and respread into selective medium containing $0.5 \mathrm{mM}$ of ouabain at a density of approximately $5 \times 10^{5}$ cells per $100-\mathrm{mm}$ dish. The selective medium was renewed on day 3. On day 7, the ouabain-resistant colonies were stained and counted. The final population sizes were corrected for plating efficiency.

Growth rates were determined according to the formula of $N_{\mathrm{t}}=N_{0} \cdot \mathrm{e}^{a t}$, where $N_{0}$ and $N_{\mathrm{t}}$ are the sizes of cell populations at time zero and $t$, and $a$ is the growth rate. A regression curve was constructed by plotting $\ln N_{\mathrm{t}}$ as the function of $t$, the 
slope of the regression curve was taken as the growth rate. The growth of the cell population was also measured by the parameter of generation time, $T$, which is a measure of the length of time required for the population size to double. $T$ was measured according to the formula: $2 N=N \cdot \mathrm{e}^{a T}$. Hence $T$ is equal to $(\ln 2) / a$.

5 out of a total of 7 available statistical methods are analyzed for the estimation of $m$. The other two methods are either too tedious in calculation or too inaccurate to be of any practical application (Armitage, 1952, 1953).

Method 1. Estimation of $m$ from the mean $(\bar{y})$ number of mutants per culture. This is Luria and Delbrück's second method. Armitage (1952) gave formulae for obtaining the estimate of $m, \hat{m}$ (Eqn. 98), and the variance of $\hat{m}$ (Eqn. 99). These two equations were followed in the present study.

Method 2. Estimation of $m$ from the upper quartile of the observed distribution of $y$. The upper quartile, $q$, is defined by Armitage (1953) as the $0.75(C+1)$ th observation when $y$ 's are arranged in increasing order, where $C$ is the total number of parallel cultures. If $C+1$ is not divisible by 4 , interpolation between adjacent values is necessary. The estimate of $m$ was calculated from Armitage's Table 3. His Eqn. 12 was followed to obtain the variance of $\hat{m}$.

Method 3. Estimation of $m$ from the median. The counts of the number of mutants in $C$ parallel cultures are arranged in ascending order and the middle culture, the $1 / 2(C+1)$ th, is called the sample median, $\hat{r}_{0}$. If $C$ is an even number, $\hat{r}_{0}$ is taken half-way between the $1 / 2 C$ th and $(1 / 2 C+1)$ th observations. The estimate of $m$ was calculated by obtaining the value of $\hat{r}_{0} / m$ from Lea and Coulson's Table 3. Their Eqn. 41 was followed to obtain the variance of $\hat{m}$.

Method 4. Estimation of $m$ from the proportion of cultures without mutants $\left(\hat{P}_{0}\right)$. This is Luria and Delbrück's first method. The observed $\hat{P}_{0}$ was substituted for $P_{0}$ to obtain $\hat{m}=-\ln \hat{P}_{0}$ (rearranged from Eqn. 5 of Luria and Delbrück, 1943). $\hat{m}$ has a variance equaling $\left(\mathrm{e}^{\hat{m}}-1\right) / N_{\mathrm{t}}$ (notation changed from Lea and Coulson's Eqn. 34).

Method 5. Estimation of $m$ via a maximum likelihood method. Detailed account of this method has been given by Lea and Coulson (1949). Laborious calculation is necessary. Briefly, the maximum likelihood estimate of $m$ is that satisfying $\Sigma\left[\left(t_{\mathrm{y}}-P_{\mathrm{y}}\right) / P_{\mathrm{y}}\right]=0$ (Lea and Coulson's Eqn. 56), where $P_{\mathrm{y}}$ is the probability of a culture having $y$ mutants and $t_{\mathrm{y}}$ is defined by Lea and Coulson's Eqn. 55. A preliminary estimate of $m$ was obtained from either the median or the $P_{0}$ method. A table where different $\left(t_{\mathrm{y}}-P_{\mathrm{y}}\right) / P_{\mathrm{y}}$ values are listed was entered at the value of $m$ nearest to this preliminary estimate. The values of $\left(t_{\mathrm{y}}-P_{\mathrm{y}}\right) / \boldsymbol{P}_{\mathrm{y}}$ were read off for each of the $C$ experimental values of $y$. The $C$ values were summed. The procedure was repeated for several adjacent values of $m$ until a value of $m$ which satisfied Eqn. 56 was found.

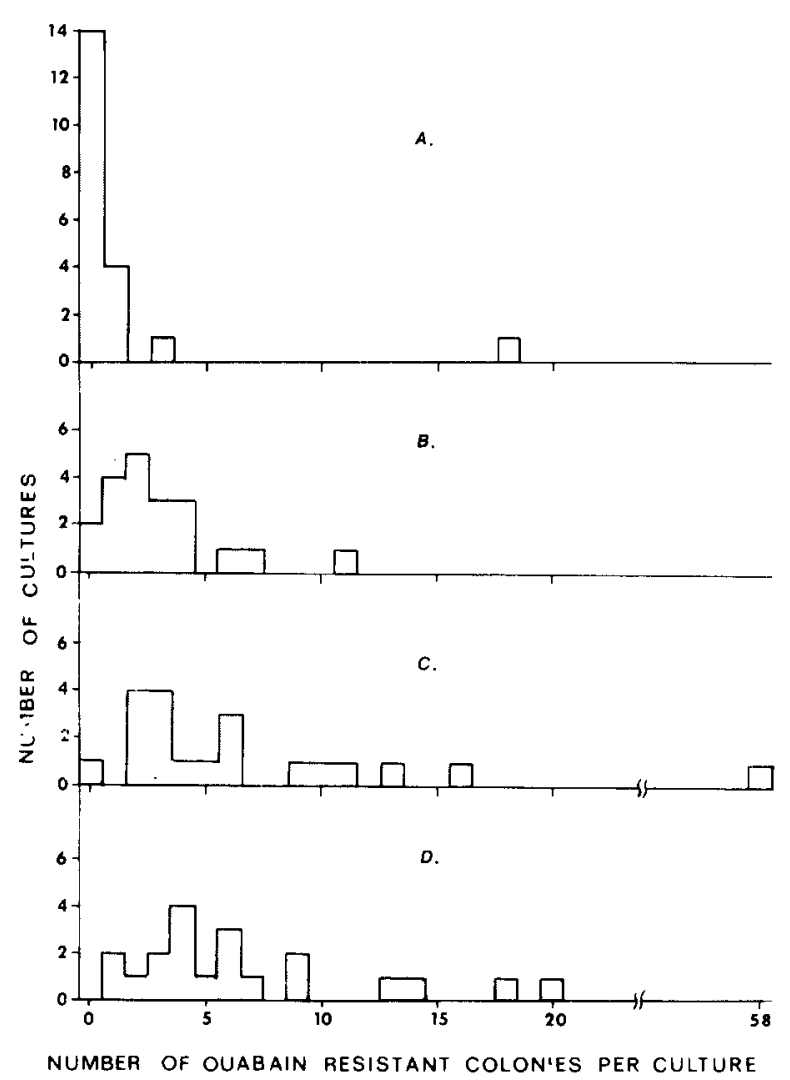

Fig. 1. Distribution patterns of the numbers of ouabainresistant mutants per culture in Chinese hamster V79 cell populations when fluctuation tests were performed with varying final population sizes. Expts. A, B, C, and D each respectively had $1.75 \times 10^{6}, 4.66 \times 10^{6}, 6.10 \times 10^{6}$, and $7.98 \times 10^{6}$ cells per culture. 
The variance of $\hat{m}$ is given by this relation $\sigma_{m}^{2}=$ $\left(m^{2} \cdot Z^{2}\right) / N_{\mathrm{t}}$ (Lea and Coulson's Eqn. 57), where $Z$ can be found from the final column of Table 4 of Lea and Coulson (1949).

After finishing the estimation of $m$, a comparison was made between the observed and expected distributions of $y$. The observed distribution was first grouped according to Lea and Coulson's Table 2, where various $P_{\mathrm{y}}$ values of $y$ are listed for different values of $m$. The expected number of cultures with a given number of $y$ were then calculated by multiplying $C$ by $P_{\mathbf{y}}$. For each set of observed distributions, there were 5 sets of expected distributions, each varied with the estimation methods of $m$ used. The sum of squared deviations, $\quad \Sigma[\text { (observed - expected)/expected }]^{2}$, or SSD, was used as an indicator of the closeness of fit between the two distributions.

\section{Results}

During the course of the experiment, culture propagation was achieved via regular cell dispersion. At the time of cell dispersion, the total cell number for each parallel culture, $N_{\mathrm{t}}$, was found to $v$ ary within a range of less than $4 \%$. The average values of the growth rates obtained from the slopes ( \pm S.E.) of regression curves of $\ln N_{\mathrm{t}}$ against $t$ were $\quad 0.0481 \pm 0.0012 / \mathrm{h}, \quad 0.00470 \pm 0.0020 / \mathrm{h}$, $0.0485 \pm 0.0008$, and $0.0460 \pm 0.0017$ respectively, for the Expts. A, B, C and D. These values are equivalent to generation times, $T$, of $14.41 \mathrm{~h}, 14.75$ $\mathrm{h}, 14.29 \mathrm{~h}$ and $15.07 \mathrm{~h}$.

The observed distributions of the number of ouabain-resistant mutants $(y)$ per culture in Chinese hamster V79 cell populations which have been grown for different lengths of time are depicted in Fig. 1. When the population size is small (Expt. A), the occurrence of mutation is infrequent; there is a large fraction of cultures containing no mutant colony. This fraction decreases as $N_{\mathrm{t}}$ increases, creating a broader distribution of $y$ (Fig. 1).

Table 1 shows a comparison of relative match

TABLE 1

COMPARISON OF THE OBSERVED AND EXPECTED DISTRIBUTIONS OF THE NUMBERS OF OUABAIN-RESISTANT MUTANTS IN Expt. C

\begin{tabular}{|c|c|c|c|c|}
\hline \multirow[t]{3}{*}{ Grouping of mutant number } & \multicolumn{4}{|c|}{ Number of cultures with the number of mutants in each grouping } \\
\hline & \multirow[t]{2}{*}{ Observed (o) } & \multicolumn{3}{|c|}{ Expected via method of a } \\
\hline & & $\begin{array}{l}\text { The mean } \\
\left(E_{1}\right)\end{array}$ & $\begin{array}{l}\text { The } P_{0} \\
\left(\mathrm{E}_{2}\right)\end{array}$ & $\begin{array}{l}\text { The maximum likelihood } \\
\left(E_{3}\right)\end{array}$ \\
\hline 0 & 1 & 3.60 & 1.00 & 1.54 \\
\hline 1 & 0 & 3.08 & 1.49 & 1.97 \\
\hline 2 & 4 & 2.35 & 1.62 & 1.92 \\
\hline $3-4$ & 5 & 3.13 & 2.97 & 3.18 \\
\hline $5-8$ & 4 & 3.11 & 4.16 & 3.97 \\
\hline $9-16$ & 5 & 2.25 & 3.92 & 3.40 \\
\hline $17-32$ & 0 & 1.27 & 2.50 & 2.06 \\
\hline $33-64$ & 1 & 1.21 & 2.34 & 1.94 \\
\hline SSD $^{b}$ & & 12.16 & 10.29 & 8.72 \\
\hline
\end{tabular}

a The expected numbers of cultures with some given number of mutants were derived by multiplying the total number of observed cultures with the theoretical probability that a culture shall have a given number of mutants (Lea and Coulson, Table 2) when the number of mutations occurring in the experiment was estimated by different methods.

${ }^{b}$ SSD, the sum of squared deviation, i.e. $\Sigma\left[\right.$ (observed - expected)/expected ${ }^{2}$, is an indicator of the match between the expected and observed distributions of the number of mutants per culture. 
between the observed distribution of $y$ from Expt. $\mathrm{C}$ and the expected distributions derived from the values of $m$ estimated by the methods of the mean, the $P_{0}$ and the maximum likelihood. As compared to the mean method, both the $P_{0}$ and the maximum likelihood methods give a better match of the two distributions.

Table 2 shows the summary of comparisons of 5 methods used for the estimation of $m$. At a smaller $N_{t}, 1.75 \times 10^{6}$, the SSDs from different methods are quite similar. However, as the $N_{\mathrm{t}}$ becomes larger, there is a larger discrepancy of the SSD values among the different methods used. Given the criterium of the smaller the SSD, the better the method, then the 'better' methods are the maximum likelihood method (No. 1), the $P_{0}$ method (No. 2), and the median method (No. 3), in that order. The 'poorer' methods are the upper quartile method (No. 4) and the mean method (No. 5). The order of preference corresponds well with the efficiency of mutation rate estimation using each method; the better methods have smaller variances of $\hat{m}$.

For the same data the $\hat{\mu}$ derived from the maximum likelihood method is approximately twice that derived from the mean method. Furthermore, as long as the population size is not too small, the maximum likelihood method gives fairly precise estimates of $\mu$ from different experiments.

\section{Discussion}

The statistical distributions of the numbers of mutants in the cell populations have been well

TABLE 2

ESTIMATION BY DIFFERENT METHODS OF THE MUTATION RATE TO OUABAIN RESISTANCE IN CHINESE HAMSTER V79 CELLS

\begin{tabular}{|c|c|c|c|c|c|c|c|}
\hline \multirow[t]{2}{*}{ Expt. } & \multirow{2}{*}{$\begin{array}{l}N_{\mathrm{t}} \\
\left(\times 10^{6}\right)\end{array}$} & \multirow[t]{2}{*}{ Parameter } & \multicolumn{5}{|l|}{ Method } \\
\hline & & & The mean & The upper quartile & The median & The $P_{0}$ & The maximum likelihood \\
\hline \multirow[t]{4}{*}{ A } & 1.75 & $\hat{m}$ & 0.38 & 0.33 & 0.29 & 0.36 & 0.34 \\
\hline & & $\hat{\mu}\left(\times 10^{-7}\right)$ & 2.18 & 1.89 & 1.66 & 2.06 & 1.94 \\
\hline & & SSD & 2.02 & 1.62 & 1.87 & 2.04 & 2.10 \\
\hline & & $\sigma_{\mathrm{m}}^{2}$ & $1.62 \times 10^{2}$ & 0.03 & $2.09 \times 10^{-7}$ & $2.45 \times 10^{-7}$ & $2.33 \times 10^{-7}$ \\
\hline \multirow[t]{4}{*}{ B } & 4.66 & $\hat{m}$ & 0.75 & 0.78 & 1.11 & 2.30 & 1.55 \\
\hline & & $\hat{\mu}\left(\times 10^{-7}\right)$ & 1.61 & 1.67 & 2.38 & 4.94 & 3.33 \\
\hline & & SSD & 22.65 & 22.06 & 22.12 & 13.34 & 10.30 \\
\hline & & $\sigma_{\mathrm{m}}^{2}$ & $7.39 \times 10^{2}$ & 0.10 & $6.44 \times 10^{-7}$ & $1.93 \times 10^{-6}$ & $6.58 \times 10^{-7}$ \\
\hline \multirow[t]{4}{*}{ C } & 6.10 & $\hat{m}$ & 1.72 & 2.03 & 2.22 & 3.00 & 2.57 \\
\hline & & $\hat{\mu}\left(\times 10^{-7}\right)$ & 2.82 & 3.33 & 3.64 & 4.92 & 4.21 \\
\hline & & SSD & 12.16 & 9.76 & 8.87 & 10.29 & 8.72 \\
\hline & & $\sigma_{\mathrm{m}}^{2}$ & $1.72 \times 10^{3}$ & 0.68 & $9.90 \times 10^{-7}$ & $3.12 \times 10^{-6}$ & $9.24 \times 10^{-7}$ \\
\hline \multirow[t]{4}{*}{ D } & 7.98 & $\hat{m}$ & 1.50 & 1.90 & 2.34 & - & 2.79 \\
\hline & & $\hat{\mu}\left(\times 10^{-7}\right)$ & 1.88 & 2.38 & 2.93 & - & 3.50 \\
\hline & & SSD & 14.69 & 9.70 & 7.24 & - & 6.76 \\
\hline & & $\sigma_{\mathrm{m}}^{2}$ & $2.59 \times 10^{3}$ & 0.28 & $1.03 \times 10^{-6}$ & - & $6.35 \times 10^{-7}$ \\
\hline
\end{tabular}

$\hat{m}$ is the estimated number of mutations occurred in the experiment.

$\hat{\mu}$ is the estimated mutation rate.

$N_{\mathrm{t}}$ is the final cell population at the time when cells were spread in ouabain medium.

$P_{0}$ is the observed proportion of cultures with no mutants.

SSD means sum squared deviation, see footnote of Table 1 for explanation.

$\sigma_{t}^{2}$ is the variance of $\hat{m}$. 
characterized by Lea and Coulson (1949), and have been expanded by Armitage $(1952,1953)$. We have simply used their statistical formulations on our experimental data to find out the best method for estimating $\mu$.

We are faced with various estimates of $m$ deduced from 5 methods. Assuming that Lea and Coulson's theoretical distribution is valid, we derived from these $m$ 's different expected distributions of $y$. The expected distributions match our data with different degree of closeness. This judgement is based on the SSD and the variance of $\hat{m}$, disregarding the possible bias each method may have when $\hat{m}$ is taken as the estimate of $m$. We $(\mathrm{Li}$ et al., 1983) have elsewhere derived the bias of the $P_{0}$ method when $P_{0}$ is substituted by $P_{0}$. The bias is around $10^{-9}$ or $10^{-10}$, too small to be of any practical importance.

When $N_{\mathrm{t}}$ is small, the distribution of $y$ is so skewed that no mutations occur. All 5 estimation methods of $m$ have to use the limited information from the clustered blocks of $y$ distribution, leaving the estimated $m$ similar. Therefore SSDs from different methods are close and no preference of methods can be made.

Owing to the tremendous sampling fluctuation in the mean, the method of using the mean number of mutants per culture for the estimate of $m$ is inaccurate, as demonstrated by the poor match between the observed and the expected distribution of mutants per culture in all 4 experiments. An objection to using this method lies in the fact that it is an extremely inefficient statistic having a large variance of $\hat{m}$. However, irrespective of what formulae we followed, either the original one of Lea and Coulson or the revised one by Armitage, the variances of $\hat{m}$ derived from the mean method are always very large. They are actually so large as to leave one doubt that both groups of authors might have committed some mistake during their derivation of their formulae.

The upper quartile method proposed by Armitage $(1952,1953)$ is also not efficient, having a variance of $\hat{m}$ in the order of $10^{-1}$. This method is based on using information of the upper quartile of the distribution with the intent of avoiding the possible effect of phenotypic delay when it occurs.

The $P_{0}$ method is highly efficient when the value of $m$ is between 0.7 and 3 (Lea and Coulson, 1949). However, the $P_{0}$ method does not provide precise information about the mutation rate when $m$ is small and the great majority of the cultures have no mutant (e.g. Expt. A). In Expts. B and C, where the values of $m$ are larger, the $P_{0}$ method provides very good match between the expected and the observed distributions of ouabain-resistant mutants. This method is easy to handle, no consultation of tables is needed. To obtain $m$, just equate $e^{-\hat{m}}$ to the proportion of cultures experimentally determined to be without mutants. However, the $P_{0}$ method is inapplicable for the determination of $m$ from experiments in which all, or nearly all, of the cultures have mutants (e.g. Expt. D). In those cases $m$ should be deduced from the median of the distribution.

The median is a highly efficient statistic with variance of $\hat{m}$ in the order of $10^{-7}$ or $10^{-6}$. The degrees of match between the observed and the expected distributions are quite good when $N_{t}$ is large (Expts. C and D). Lea and Coulson (1949) provided a table for the estimation of $m$ by this method.

The maximum likelihood method is the best method to use for the derivation of $m$ in the 4 fluctuation experiments we have presented, as judged by the smallest variance of $m$ and the closest match between the observed and the expected distributions of ouabain-resistant mutants. This method is statistically fully efficient but needs laborious computation. In our case, the value of $m$ for each experiment was deduced through a procedure of repeating calculations. The $m$ so obtained maximized the likelihood of the sample result, as manifested by the smallest SSD values for each experiment. When cell population sizes are large, the $\hat{\mu}$ 's obtained from the maximum likelihood method are quite precise. This confirms the notion that $\mu$ is a constant parameter of a cell line as long as the cell population is maintained at its logarithmic phase of growth, with each cell having the same chance to mutate. 


\section{Conclusion}

The mean and the upper quartile methods should be abandoned. There are advantage and disadvantage for each of the $P_{0}$ and the median methods. We hope the labor of obtaining an estimate of $m$ from the maximum likelihood method will not be prohibitive to biologists, because this method is the best choice either theoretically or experimentally.

The values of $\hat{\mu}$ presented in Table 2 seem to vary only within a factor of 3 , depending on the size of $N_{\mathrm{t}}$ and the method for estimation of $m$. However, the variation could become substantially larger when the method used is accompanied by inappropriate experimental design (Fu et al., 1982; Li et al., 1983).

In conclusion, we suggest that for the stochastic approach of mutation studies with cultured mammalian cells, the combined effects of the method of estimation of $\mu$, the final population size, the growth condition, and the experimental design should be carefully considered so that a reliable estimate of $\mu$ could be achieved.

\section{Acknowledgements}

We thank Dr. Yung-Tai Hung for consultation on statistics, Drs. Wen-hsiung Robert Tseng, Peter Smouse for reading the manuscript.

\section{References}

Armitage, P.J. (1952) The statistical theory of bacterial populations subject to mutation, J. Roy. Statist. Soc., B14, 1-40.

Armitage, P.J. (1953) Statistical concepts in the theory of bacterial mutation, J. Hyg., 51, 162-184.

Fu, J.L., L.C. Li and E.H.Y. Chu (1982) The parameters for quantitative analysis of mutation rates with cultured mammalian somatic cells, Mutation Res., 105, 363-370.

Lea, D.E., and C.A. Coulson (1949) The distribution of the numbers of mutants in bacterial populations, J. Genet., 49 , 264-285.

Li, I.-C., J. Fu, Y.-T. Hung and E.H.Y. Chu (1983) Estimation of mutation rates in cultured mammalian cells, Mutation Res., 111, 253-262.

Li, I.-C., S.-C. Ho Wu, J. Fu and E.H.Y. Chu (1985) A deterministic approach for the estimation of mutation rates in cultured mammalian cells, Mutation Res., 149, 127-132.

Luria, S.E., and M. Delbrück (1943) Mutations of bacteria from virus sensitivity to virus resistance, Genetics, 28 , 491-511.

Communicated by T. Sugimura 